\title{
Comparative and temporal transcriptome analysis of peste des petits ruminants virus infected goat peripheral blood mononuclear cells
}

Siddappa

smanju712@gmail.com

Bishnu Prasad Mishr

bpmishra_1@hotmail.com

Bina Mishra

binachauhanmishra@hotmail.com

Aditya Prasad Sahoo

rush2aditya@gmail.com

Ashok K. Tiwari

aktiwari63@yahoo.com

Kaushal Kishore

kaushalvirol@gmail.com

D. Muthuchelvan

drchelva@gmail.com

Shikha Sax

shikhasaxenaivri@gmail.com

Lakshman Santra

lakshman.ivri@gmail.com

Amit Ranjan Sahu

dramitr.sahu@gmail.com

Sajad Ahmad

wanisajad759@gmail.com

R.P. Singh ${ }^{d}$

rpsingh@dr.com

Y.P. Singh

ypivri@gmail.com

Aruna Pandey

arunabioinfo@gmail.com

Sonam Kanchan

kanchansonam@gmail.com

R.K. Singh

This is the author's manuscript of the article published in final edited form as:

Manjunath, S., Mishra, B. P., Mishra, B., Sahoo, A. P., Tiwari, A. K., Rajak, K. K., ... Janga, S. C. (2017). Comparative and temporal transcriptome analysis of peste

des petits ruminants virus infected goat peripheral blood mononuclear cells. Virus Research, $229,28-40$. https://doi.org/10.1016/j virusres.2016.12.014 


\section{Gandham Ravi Kumar ${ }^{a}$ *}

gandham71@gmail.com

Sarath Chandra Janga ${ }^{\mathrm{f}, \mathrm{g}, \mathrm{h}, * *}$

scjanga@iupui.edu

a Division of Veterinary Biotechnology, ICAR-Indian Veterinary Research Institute, Izatnagar, Bareilly, Uttar Pradesh, 243122, India

${ }^{\mathbf{b}}$ Division of Biological Standardization, ICAR-Indian Veterinary Research Institute, Izatnagar, 243122, India

'Division of Virology, ICAR-Indian Veterinary Research Institute (IVRI), Mukteswar Campus, Nainital, Uttaranchal, 263138, India

dDivision of Biological Products, (ICAR-)Indian Veterinary Research Institute, Izatnagar, Bareilly, Uttar Pradesh, 243122, India

eARIS Cell, (ICAR-Indian Veterinary Research Institute (IVRI), Mukteswar Campus, Nainital, Uttaranchal, 263138, India

fSchool of Informatics and Computing, Indiana University Purdue University, 719 Indiana Ave Ste 319, Walker Plaza Building, Indianapolis, IN 46202, United States

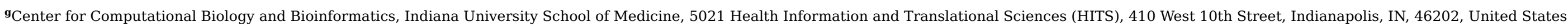

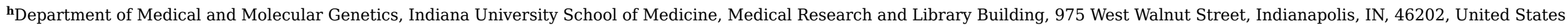

${ }^{*}$ Corresponding author.

${ }^{* *}$ Corresponding author at: School of Informatics and Computing, Indiana University Purdue University, 719 Indiana Ave Ste 319, Walker Plaza Building, Indianapolis, IN 46202, United States.

(Delete the this line )1 ARIS Cell, Indian Veterinary Research Institute, Izatnagar, Bareilly, Uttar Pradesh, 243122, India.

\section{Abstract}

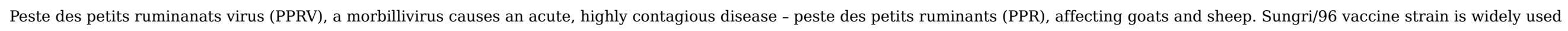

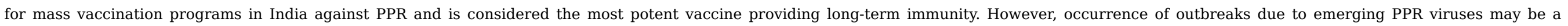

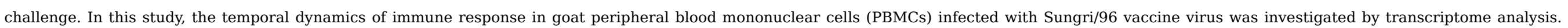

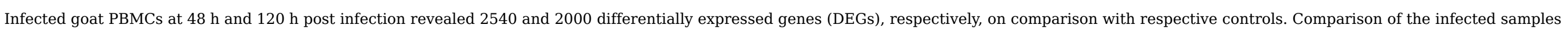

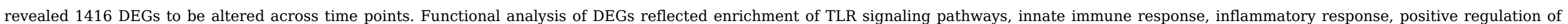

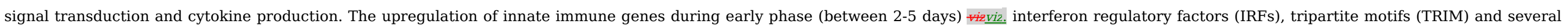

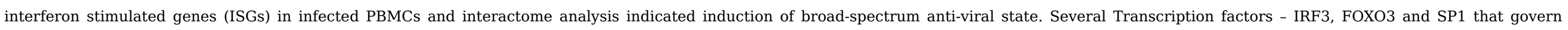

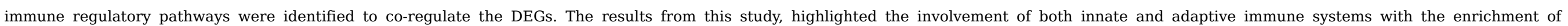

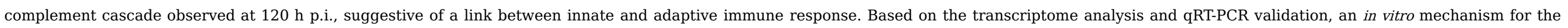
induction of ISGs by IRFs in an interferon independent manner to trigger a robust immune response was predicted in PPRV infection.

Keywords: PPRV; PBMCs; RNA-sequencing; Transcriptome; Protein-protein interaction networks; Transcription factors

\section{Introduction}




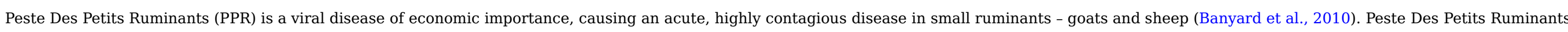

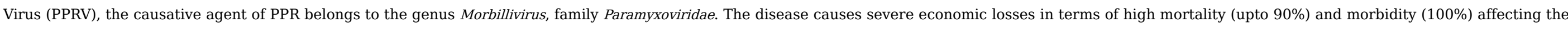

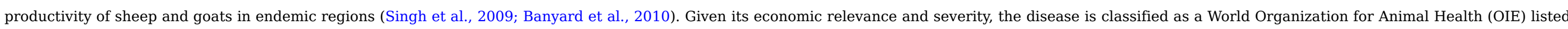

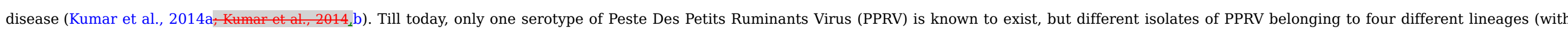

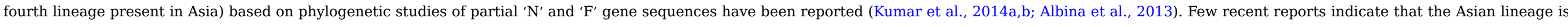

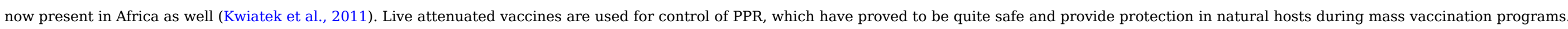
Among these live attenuated vaccines, Nigeria 75/1 and Sungri/96 have been widely used for the control of PPRV in Africa (Diallo et al., 1989) and India (Saravanan et al., 2010) respectively.

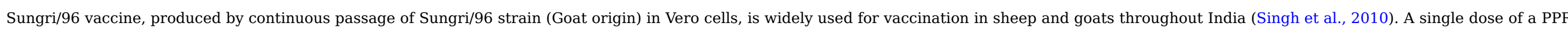

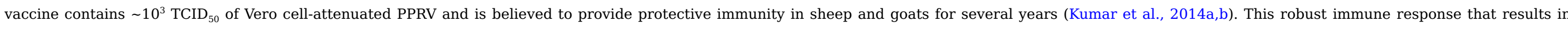

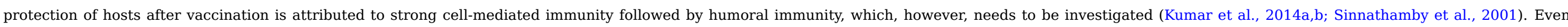

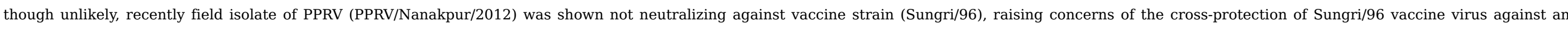
emerging field virus (Kumar et al., 2014a,b), and making it all the more important to understand the complete mechanisms of immune protection induced by Sungri/96 PPRV vaccine strain.

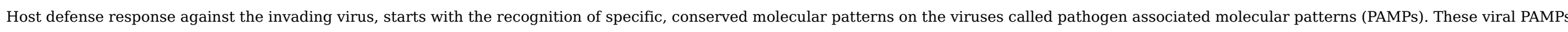

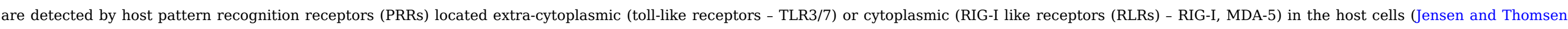

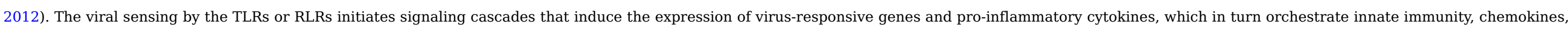

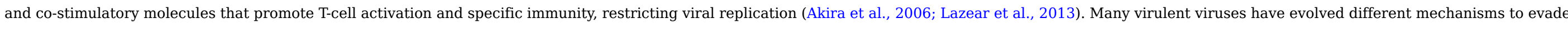

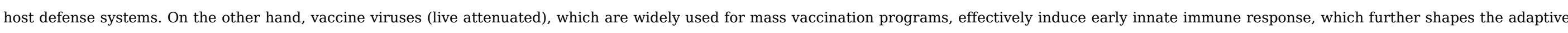
immunity giving a robust immune response (Kumar et al., 2014a,b).

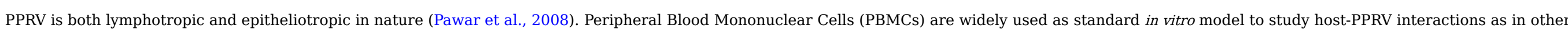

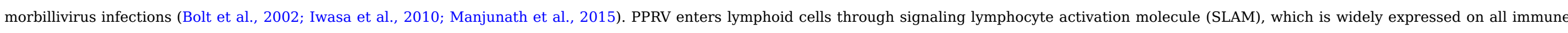

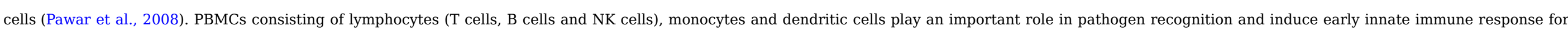

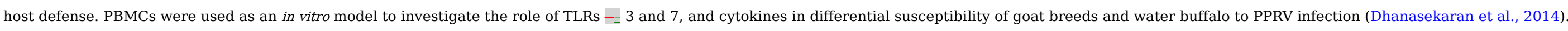

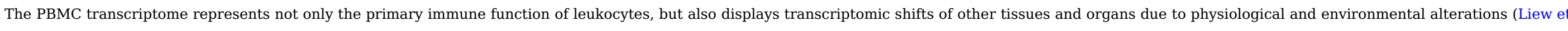

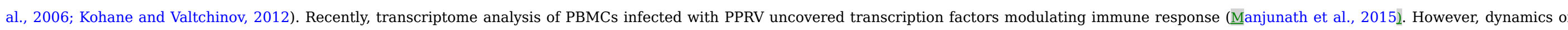

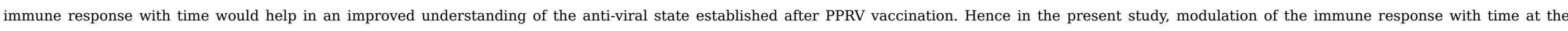

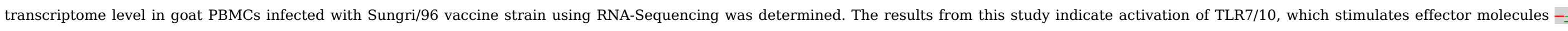
interferon stimulated genes inia interferon regulatory factors in interferon independent manner to provide an effective anti-viral response against PPRV infection.

\section{Material and methods}

\subsection{Ethics statement and animals}

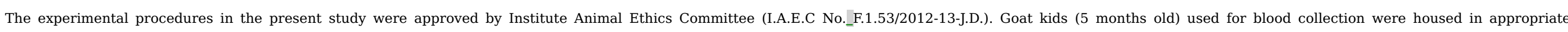
containment facilities with feed and water ad libitum.

\subsection{Virus propagation and purification}

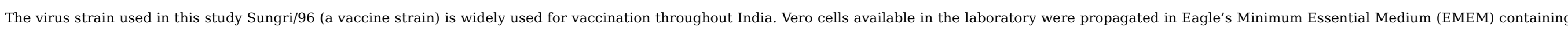

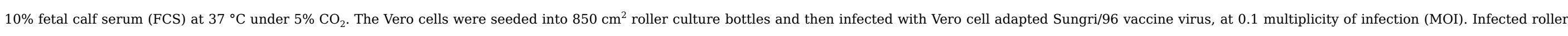

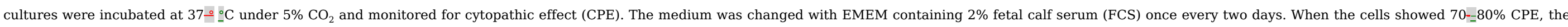




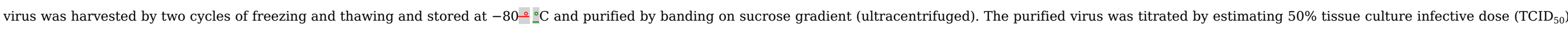
using Vero cells in 96 well microtitre plate. The purified virus was tested for its infectivity in Vero cells and was used further for infection in goat PBMCs.

\subsection{Screening of animals for PPRV antibodies}

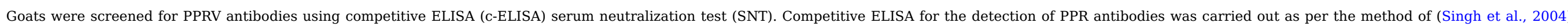
using c-ELISA Kit (IVRI, Mukteshwar). PBMCs were isolated from blood collected from animals that showed SNT titre $>1: 8$ and Percentage Inhibition (PI) value less than 40\%.

\subsection{Virus infection in PBMCs with PPRV and confirmation}

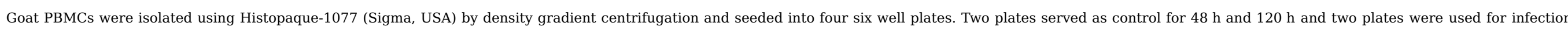

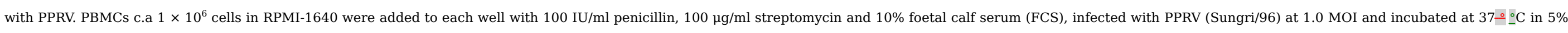

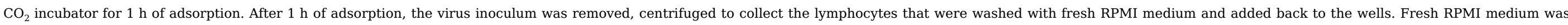

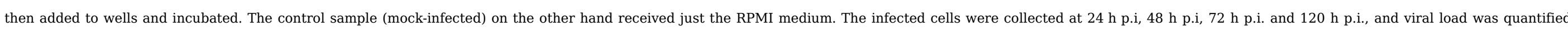

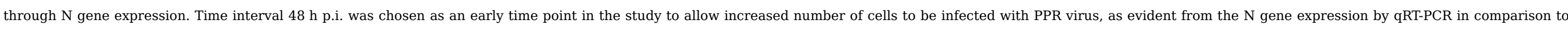

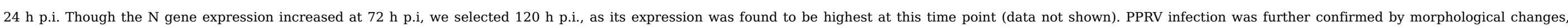
reverse transcription polymerase chain reaction (RT-PCR) (Manjunath et al., 2015) and FACS at both 48 and $120 \mathrm{~h}$ p.i.

\subsection{Flow cytometry analysis of PPRV infected PBMCs}

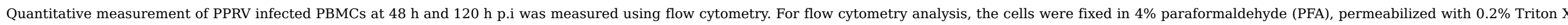

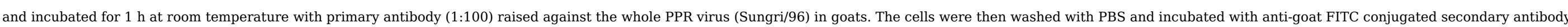

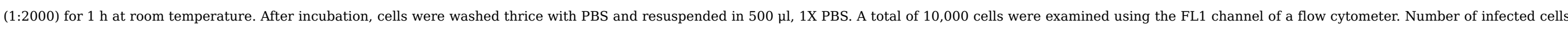
(PPRV positive PBMCs) at $48 \mathrm{~h}$ and $120 \mathrm{~h}$ p.i. were analyzed using CellQuest software (BD Biosciences).

\subsection{RNA extraction, library preparation and Illumina sequencing}

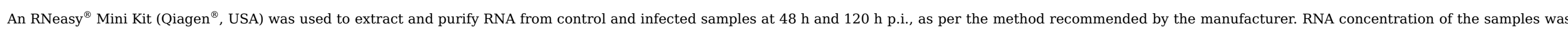

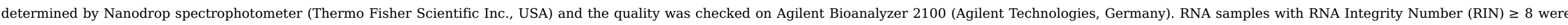

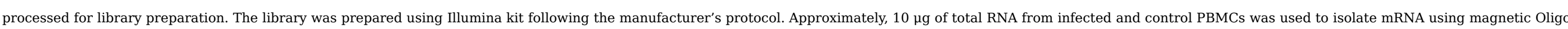

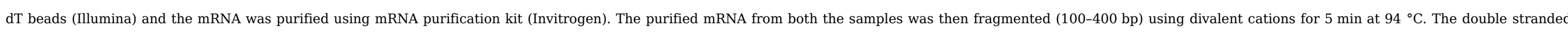

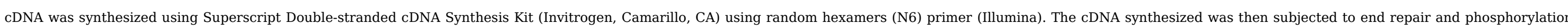

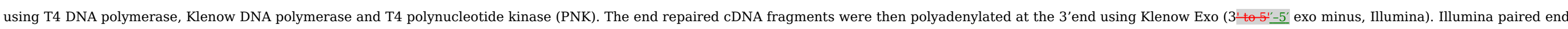

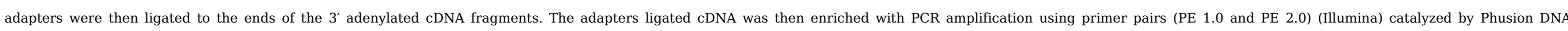

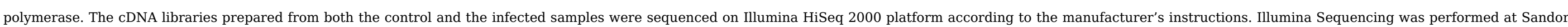
LifeSciences, Pvt. Ltd_ (Hyderabad, India).

\subsection{Raw data preprocessing}

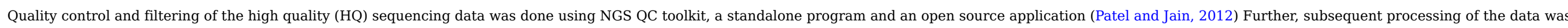

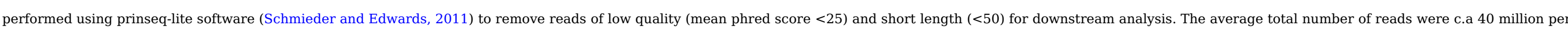

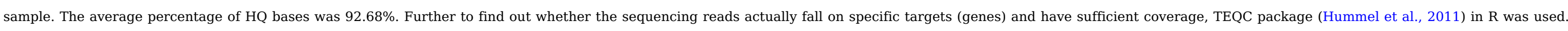
The coverage and normalized coverage of five genes at both the time points is given in Fig. 1. 


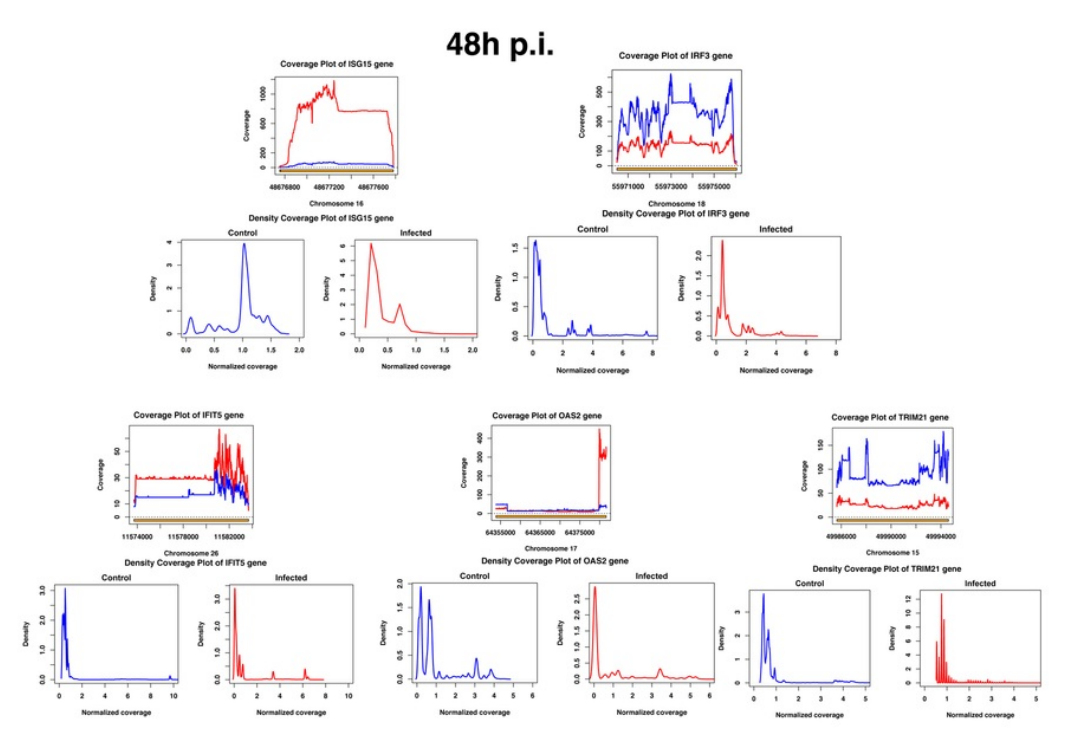

120h p.i.
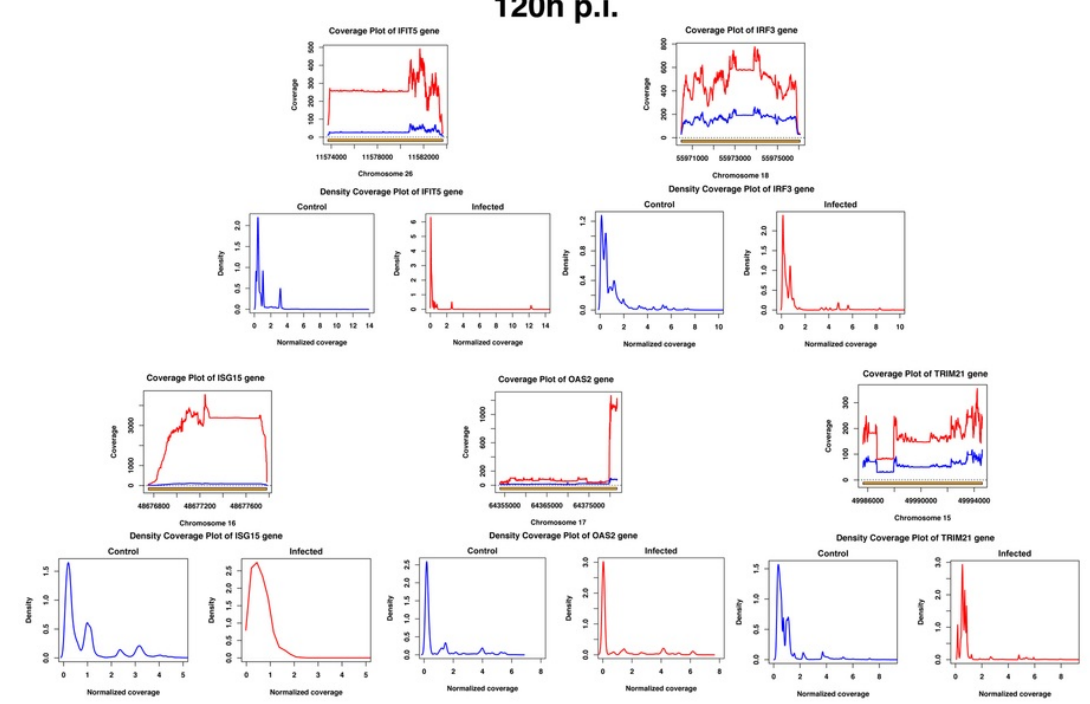

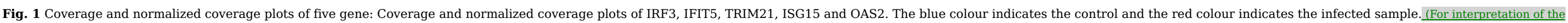
references to colour in this figure legend, the reader is referred to the web version of this article.

alt-text: Fig. 1

\subsection{Viral transcript quantification}

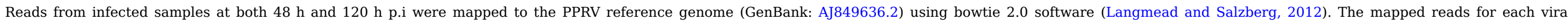

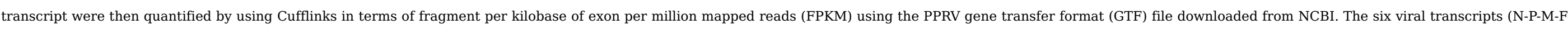




\subsection{Identification of differentially expressed genes (DEGs) on comparative and temporal analysis and functional annotation}

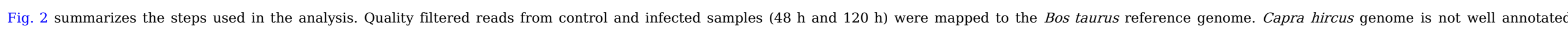

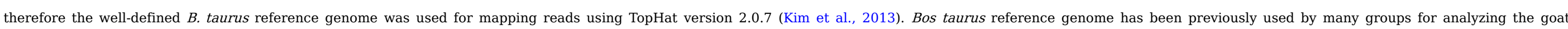

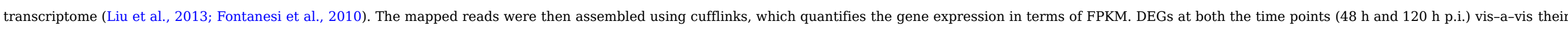

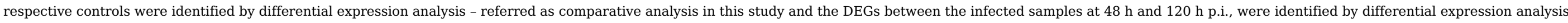

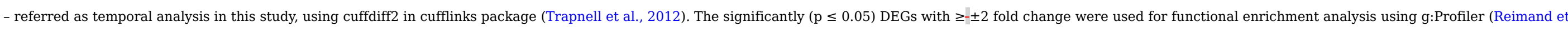

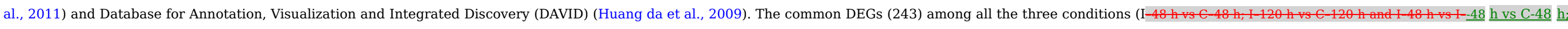

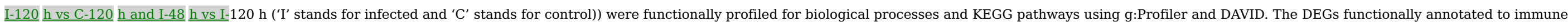
processes and common DEGs were further subjected to ClueGO analysis (Bindea et al., 2009) for functional enrichment in KEGG and REACTOME. 


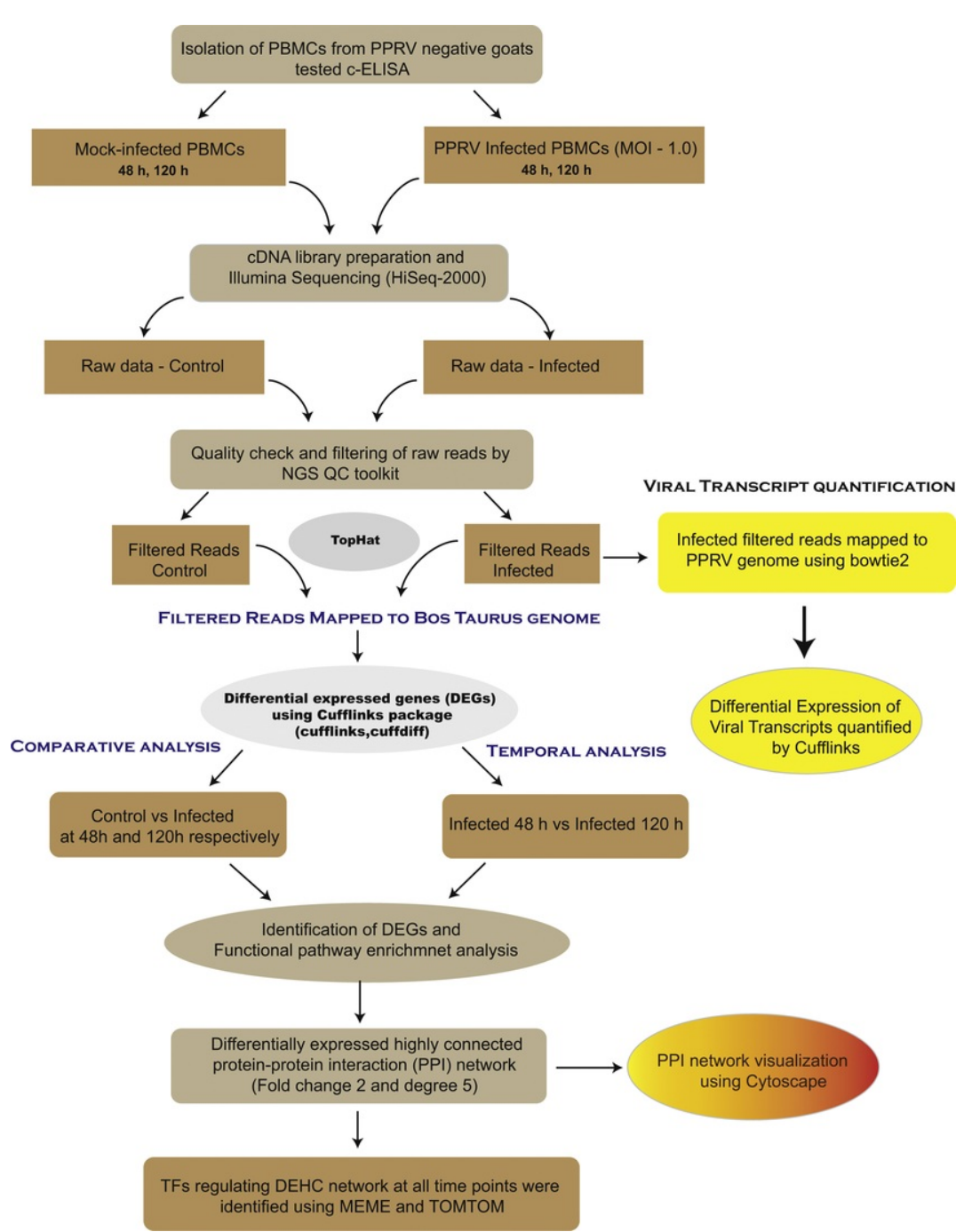

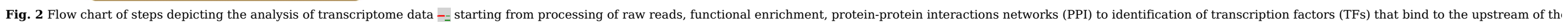
differentially expressed highly connected (DEHC) genes from comparative and temporal data analysis.

alt-text: Fig. 2

\subsection{Protein-Pprotein interaction (PPI) networks of differentially expressed and highly connected genes (DEHC)}

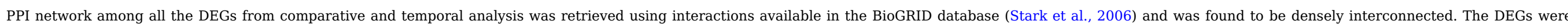

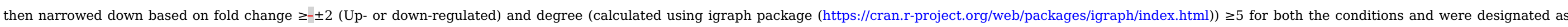

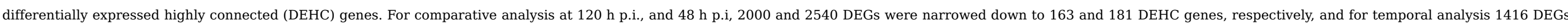
were narrowed down to 126 DEHC genes. The PPI network for DEHC genes was visualized using Cytoscape software (Shannon et al., 2003).

\subsection{Identification of transcription factors (TFs) regulating DEGs}




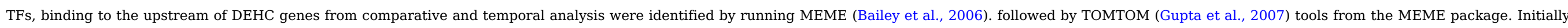

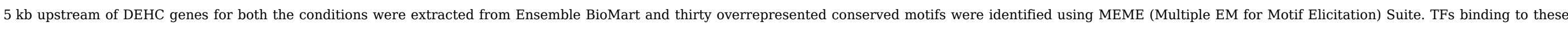

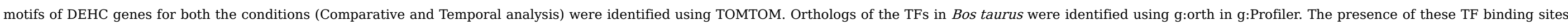
across the DEHC genes areis shown as a heatmap using geneE software (Broad Institute).

\subsection{Validation of RNA sequencing data using Quantitative Real time PCR (qRT-PCR)}

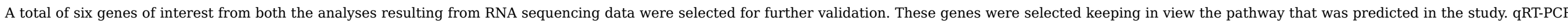

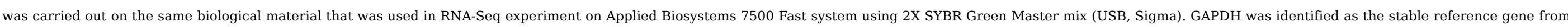

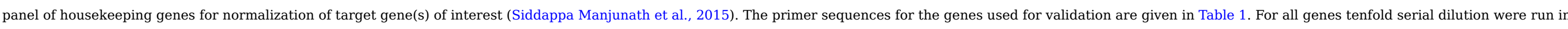

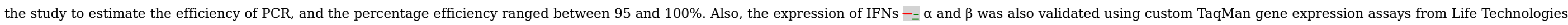

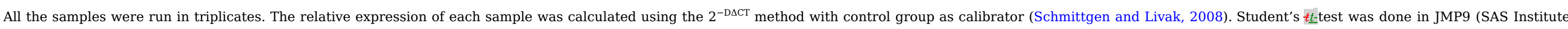
Inc, Cary, USA) and differences between groups were considered significant at $\mathrm{p} \leq 0.05$.

Table 1 Genes and their primer sequences used for validation of the RNA-sequencing data and prediction of immune signaling pathway.

\section{alt-text: Table 1}

Genes

Primer sequence

Accession Numbers

IRF3

Forward: AGCGTCCCTAGCAGACAAGA

Reverse: CCAGGTTGAACACACCTCCT

$$
\text { ISG15 }
$$

Forward: CAGTTCATCGCCCAGAAGAT Reverse: GTCGTTCCTCACCAGGATGT

\begin{tabular}{|l|l|}
\hline HERC5 & Forward: GTATGAGGTTGGCTGGCATT
\end{tabular}
Reverse: CCCTGACTCCTCCAAAATCA

\begin{tabular}{l|l}
\hline IFIT3 & Forward: AAGGGTGGACACTGGTCAAG
\end{tabular}
Reverse: AGGGCCAGGAGAACTTTGAT

\begin{tabular}{l|l} 
IFIT5 & Forward: CTTGGAGGTGACACCAACCT
\end{tabular} Reverse: CCACAGCTGCTTTGAAATGA

IRF7 Forward: GACACGCCCATCTTTGACTT Forward: GACACGCCCATCTTTGACTT
Reverse: ACTGTCCAGGGAGGACACAC

\begin{tabular}{|l|l}
\hline Primer sequence & JQ308793.1 \\
\hline & XM_005690795.1 \\
\hline & XM_005681669.1 \\
\hline & XM_005698196.1 \\
\hline & XM_005698239.1 \\
\hline & XM_004019737.2
\end{tabular}

\section{Results}

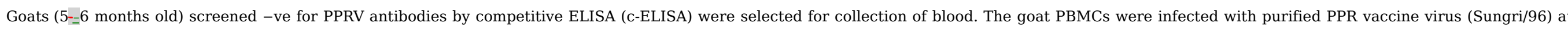

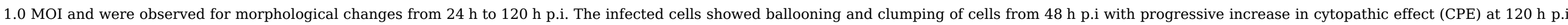

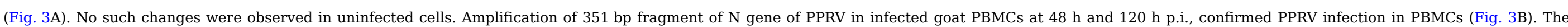

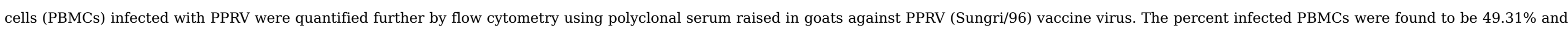

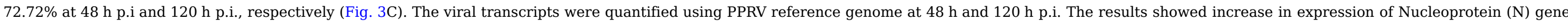
followed by fusion (F), Haemagglutinin (H), Large (L), Matrix (M), and Phosphoprotein (P) with time (Fig. 3D). These results ratified progression of PPRV infection from $48 \mathrm{~h}$ to $120 \mathrm{~h}$ p.i. 

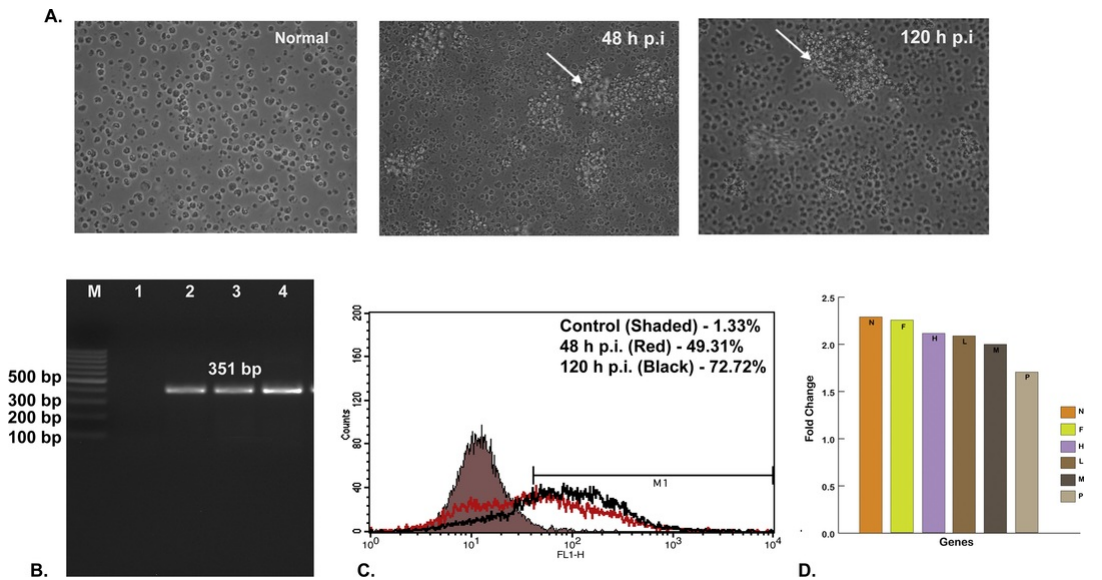
c.

D.

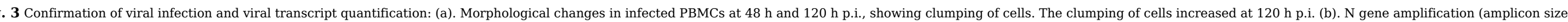

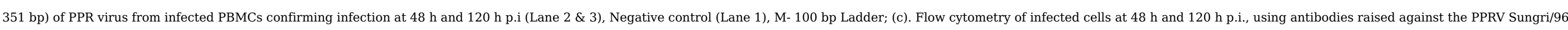

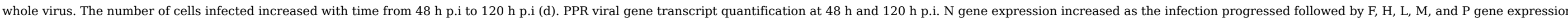
alt-text: Fig. 3

\subsection{Identification of DEGs on comparative and temporal analysis and functional annotation}

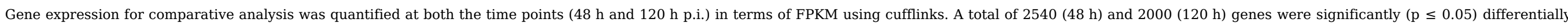

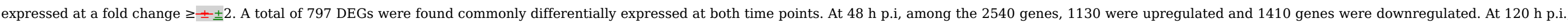

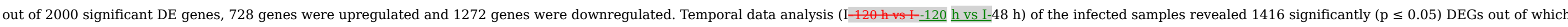
821 genes were upregulated and 595 genes were downregulated with fold change $\geq \pm \pm 2$.

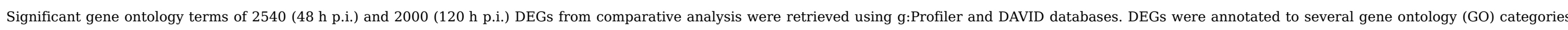

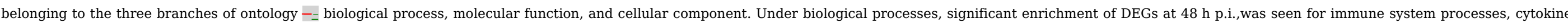

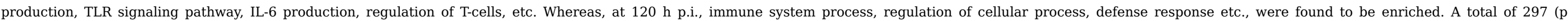

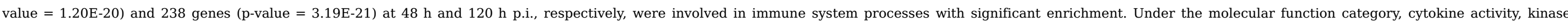

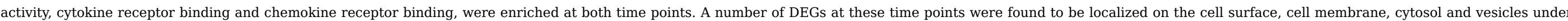

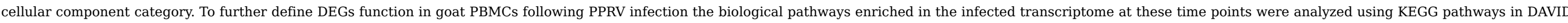

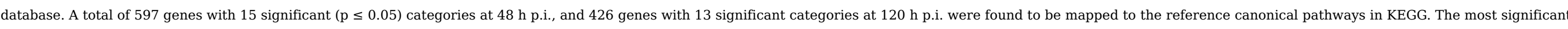

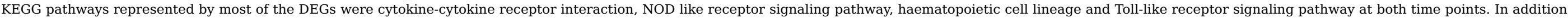
enrichment of complement cascade was observed at $120 \mathrm{~h}$ p.i. suggesting it as a link between innate and adaptive response.

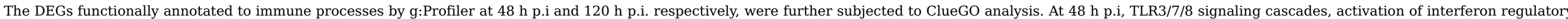

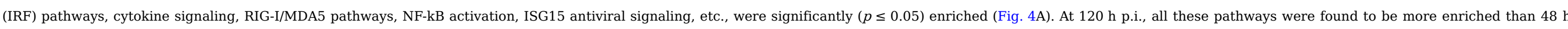

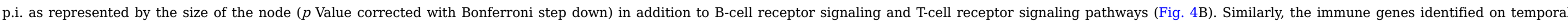

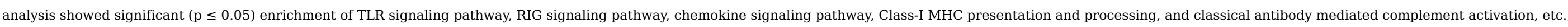
(Fig. 4C). The enrichment of these pathways in temporal analysis clearly indicated that the immune response was progressing from innate to adaptive with time. 


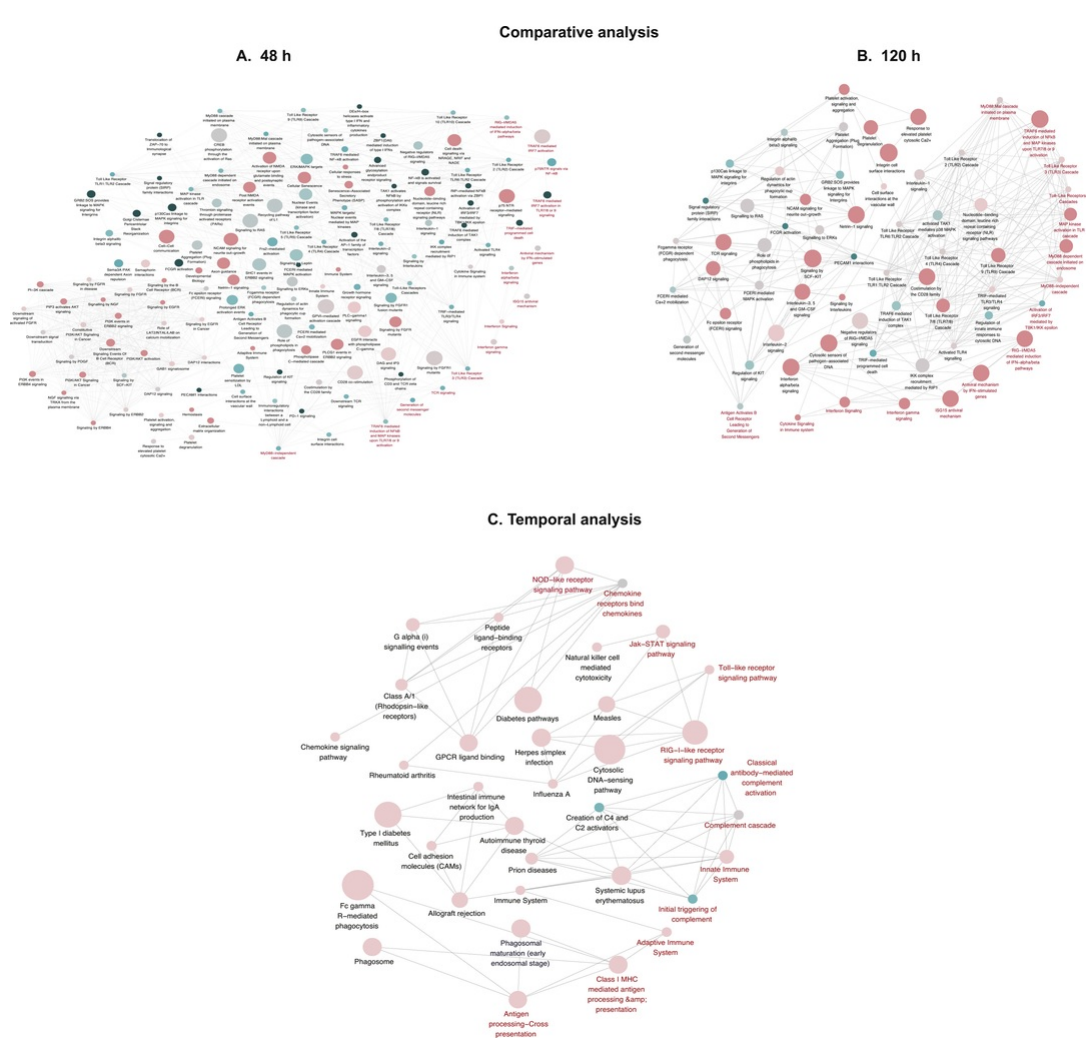

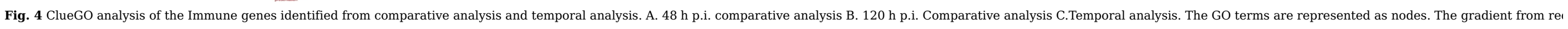

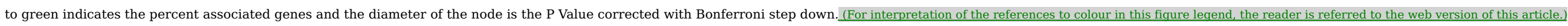
alt-text: Fig. 4

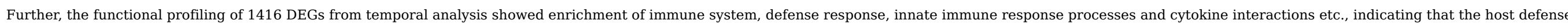

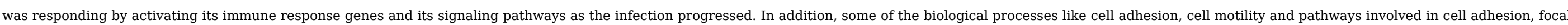
adhesions, adherens junctions, etc., were enriched indicating that the cytoskeleton and cell to cell communications were affected as PPRV infection progressed.

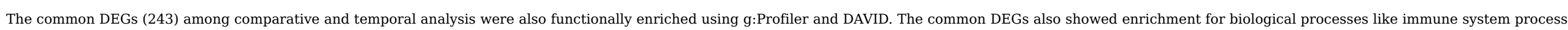

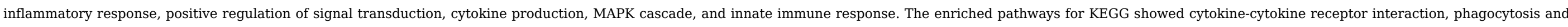

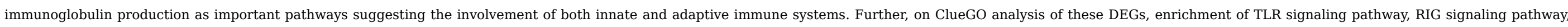

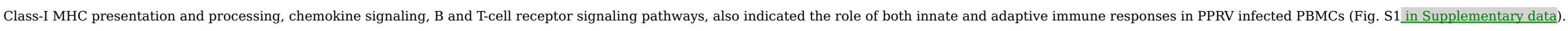

\subsection{DEGs and broad spectrum anti-viral response}

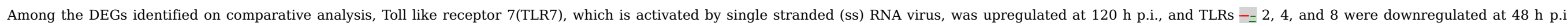

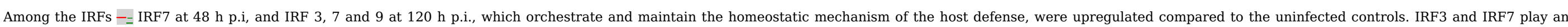

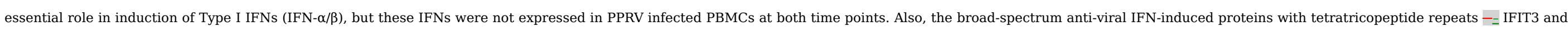

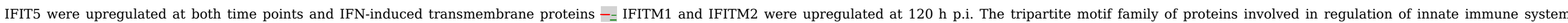




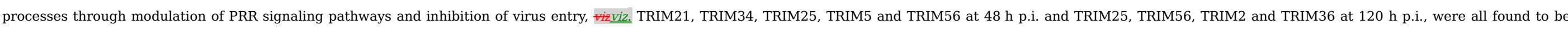

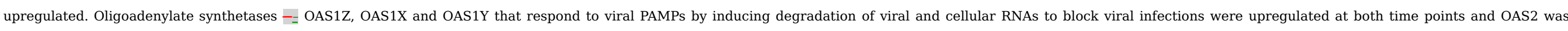

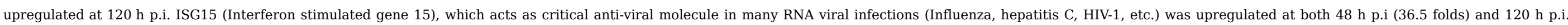

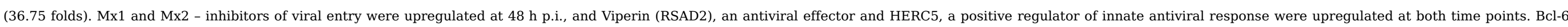

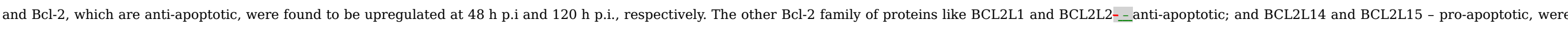

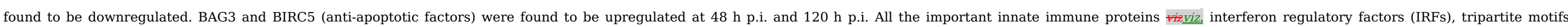
(TRIMs) and interferon stimulated genes (ISGs), which act as first line of host defense against the invading viruses were all found to be significantly upregulated at both time points.

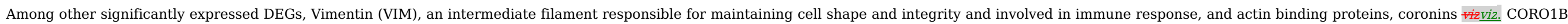

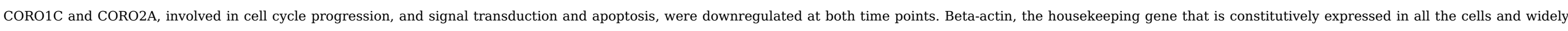

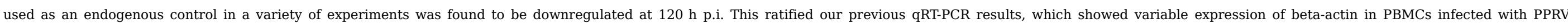
(ㅡanjunath et al., 2015). This suggested downregulation of cytoskeletal proteins due to PPRV infection in the infected PBMCs.

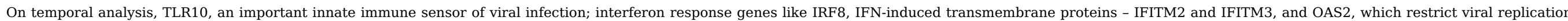

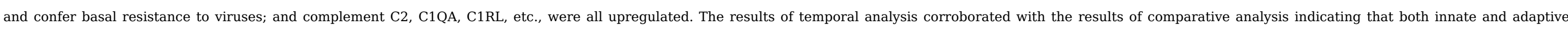
immune system were activated to establish an anti-viral state as PPRV infection progressed.

\subsection{Protein-protein interaction network of DEHC genes}

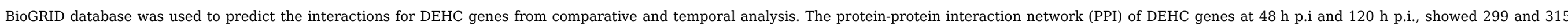

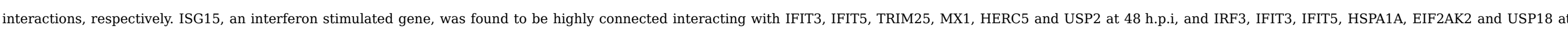

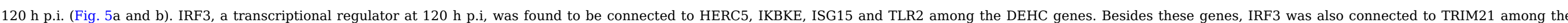

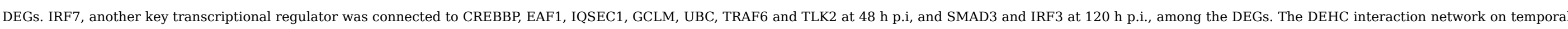

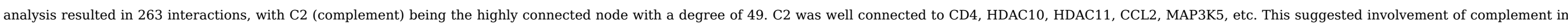

linking adaptive and innate immunity as the PPRV infection progressed with time (Fig. 5c). 

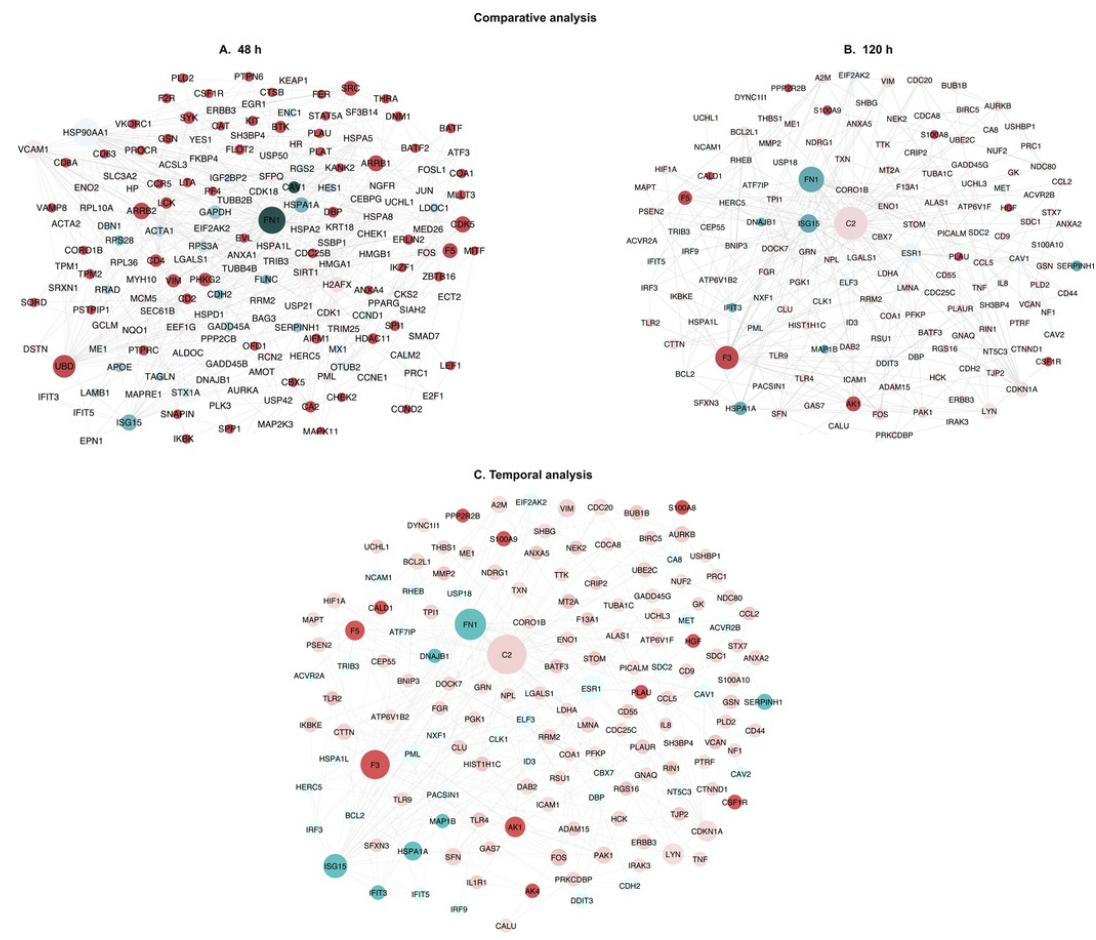

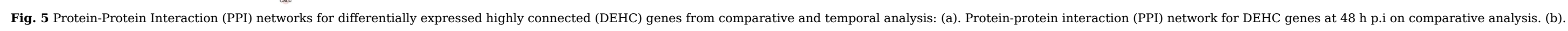

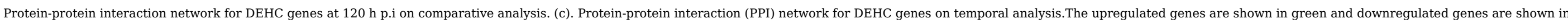

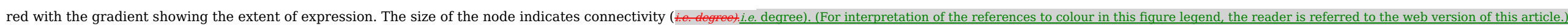

\section{alt-text: Fig. 5}

\subsection{Transcription factors co-regulating the DEHC genes}

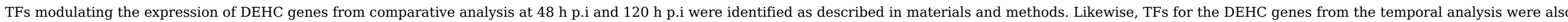

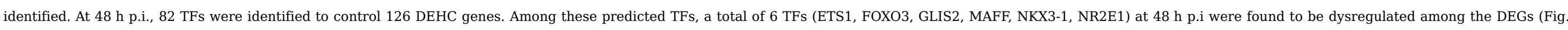

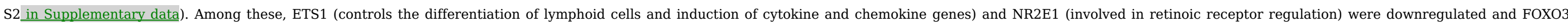

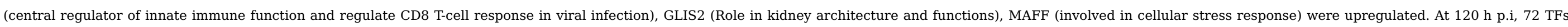

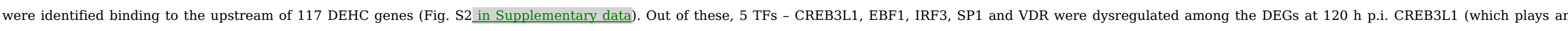

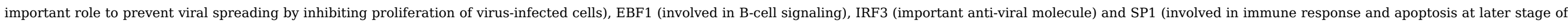

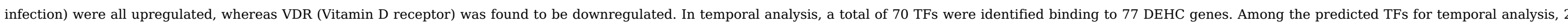

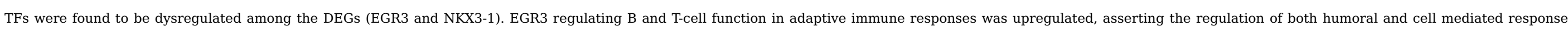
genes as infection progressed (Fig. S3 3 in Supplementary data).

\subsection{Validation of RNA sequencing data qRT-PCR}

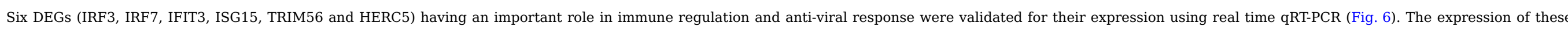
DEGs was in concordance with RNA sequencing results. The expression of IFNs - $\alpha$ and $\beta$ was found to be non - significant at both the time points (Fig. S4 in Supplementary data). 


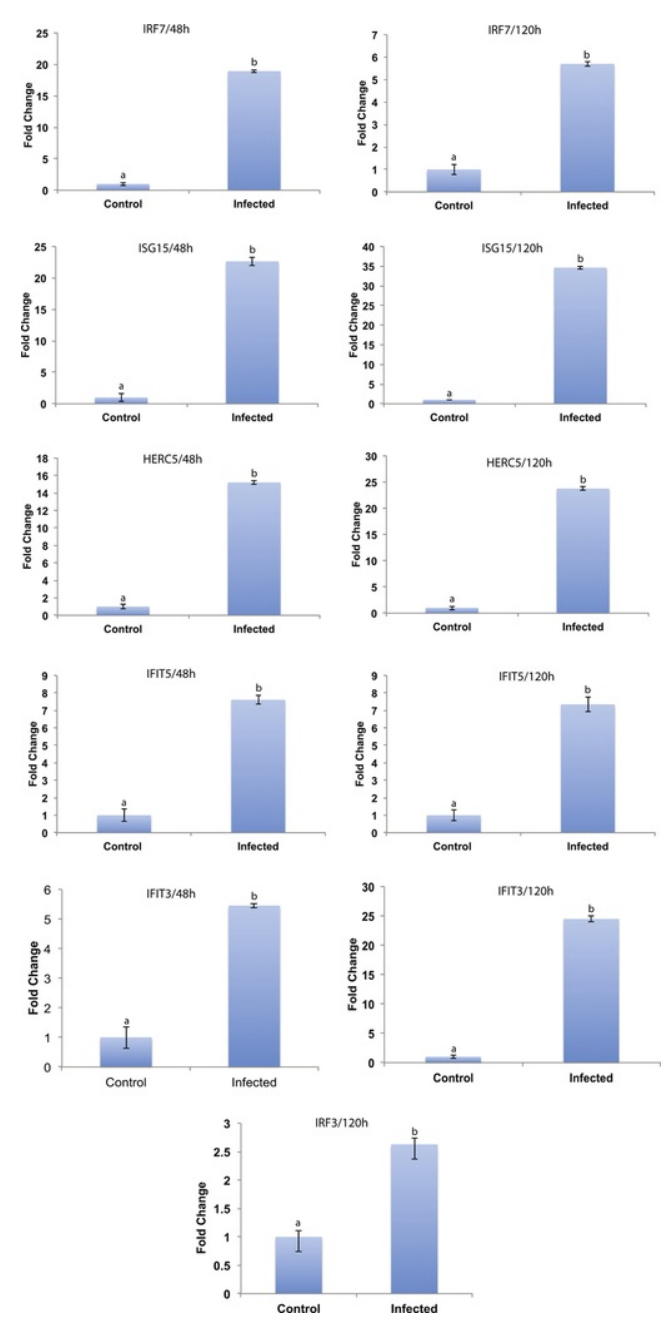

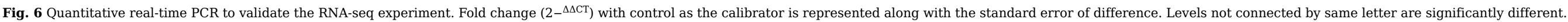
alt-text: Fig. 6

\section{Discussion}

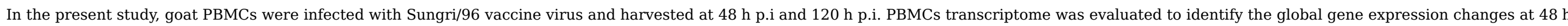
p.i. and $120 \mathrm{~h} \mathrm{p.i,} \mathrm{to} \mathrm{understand} \mathrm{and} \mathrm{delineate} \mathrm{the} \mathrm{mechanism} \mathrm{of} \mathrm{early} \mathrm{immune} \mathrm{protection} \mathrm{induced} \mathrm{by} \mathrm{the} \mathrm{vaccine} \mathrm{virus.}$

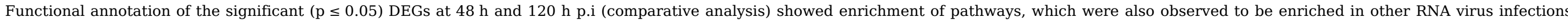

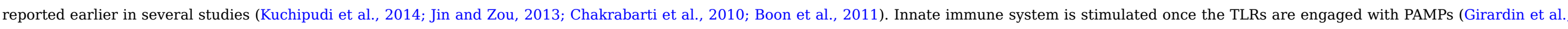

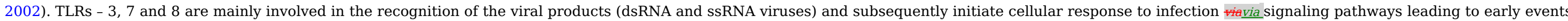

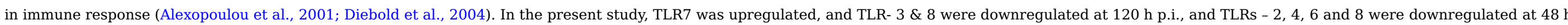

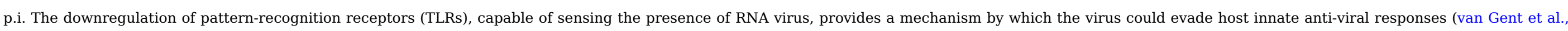




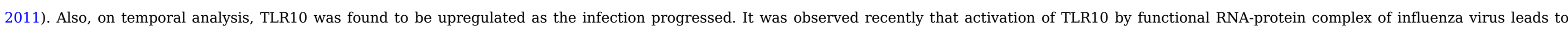
robust induction of cytokine expression (Lee et al., 2014). Thus, in PPRV infection TLR7 and TLR10 may play an important role in triggering the downstream immune signaling networks.

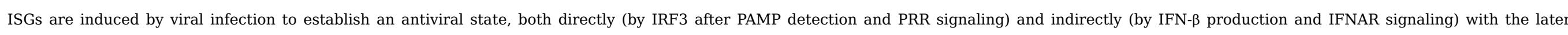

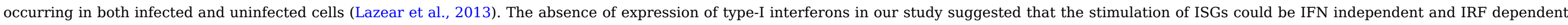

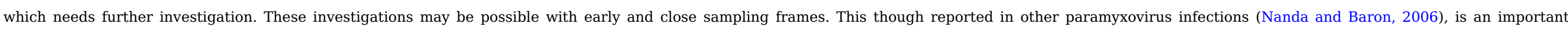

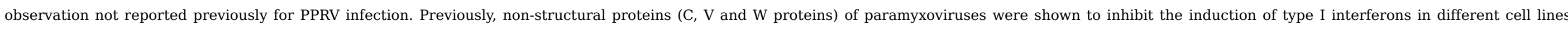

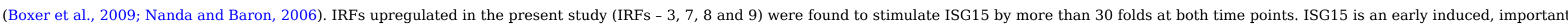

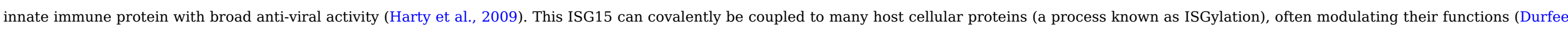

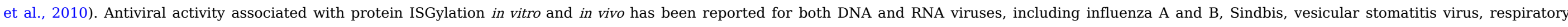

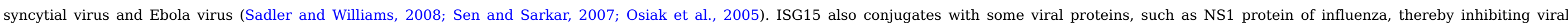

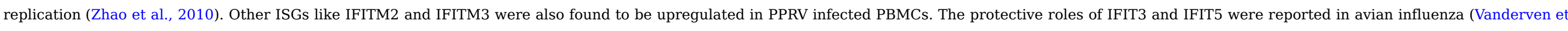
al., 2012) dengue virus (Hsu et al., 2013) and other RNA virus infections thus, reasserting their possible role in PPRV infection.

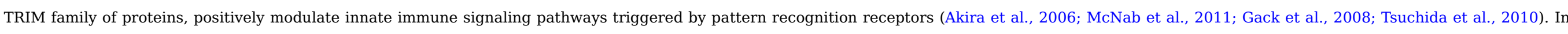

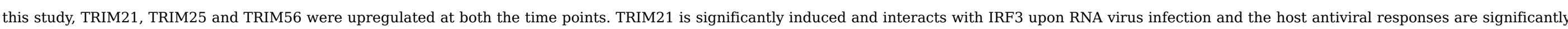

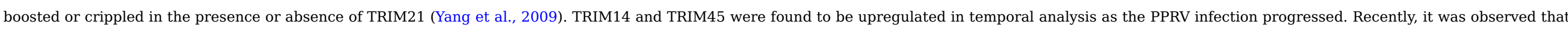

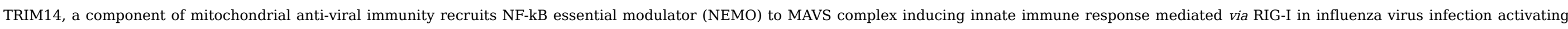

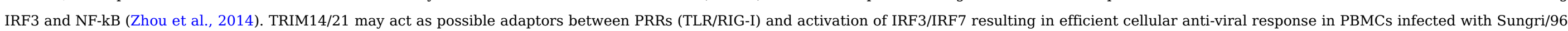
PPRV vaccine virus.

\subsection{Predicted Immune signaling pathway triggered by Sungri/96 vaccine virus}

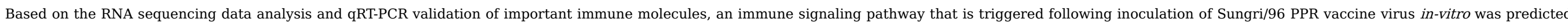

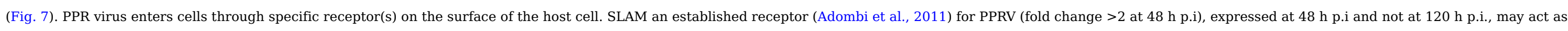

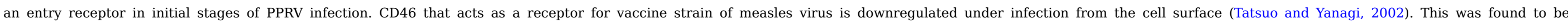

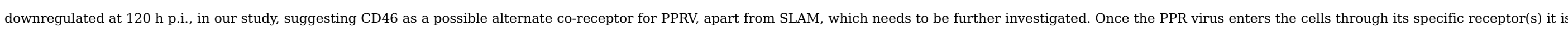

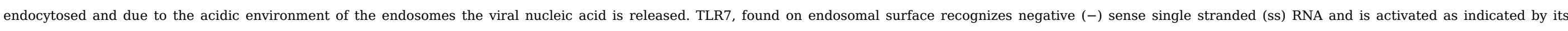

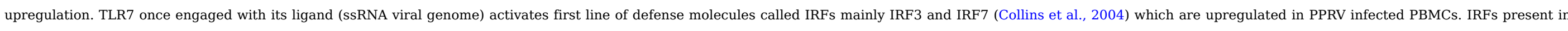

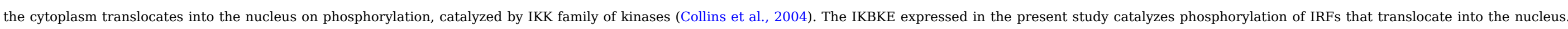

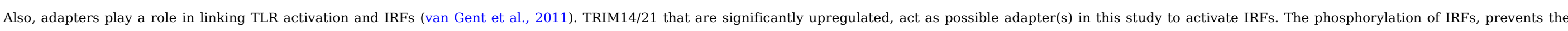

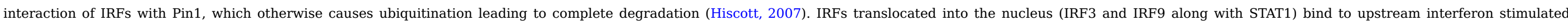

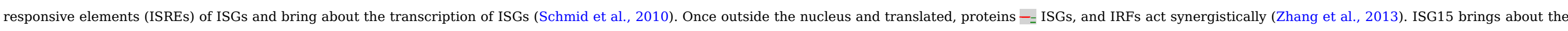

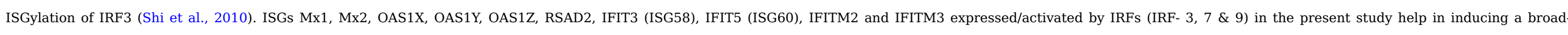

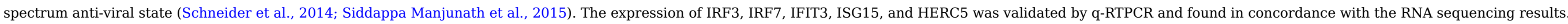




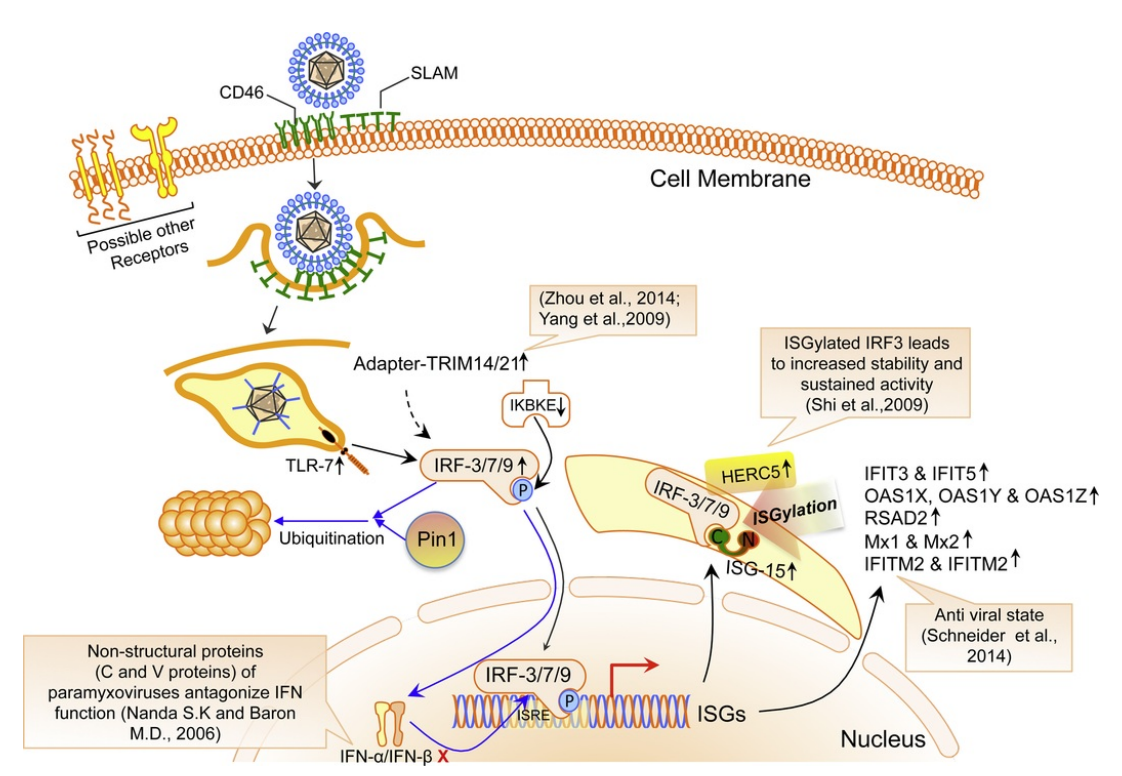

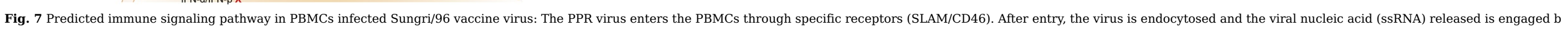

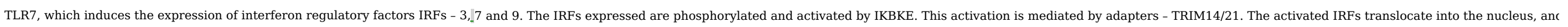

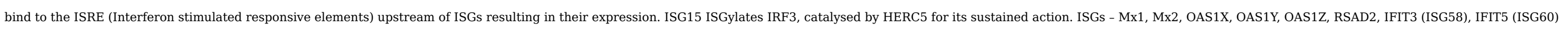

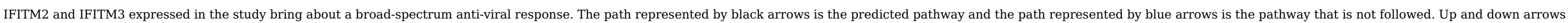

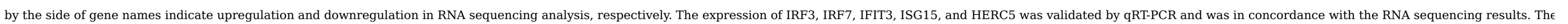
crossmark in red indicates absence of expression. (For interpretation of the references to colour in this figure legend, the reader is referred to the web version of this article.)

\section{alt-text: Fig. 7}

\section{Conclusion}

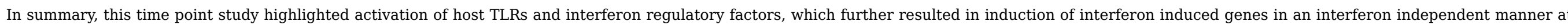
early time points. Anti-viral response induced by Sungri/96 involved both innate and adaptive immune systems with the enrichment of complement cascade observed at $120 \mathrm{~h}$ p.i.

\section{Conflict of interest}

The authors declare no conflict of interest.

\section{Acknowledgements}

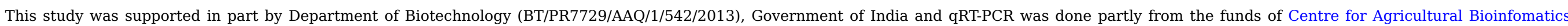
(ICAR-IASRI)

\section{Appendix A. Supplementary data}

Supplementary data associated with this article can be found, in the online version, at http://dx.doi.org/10.1016/j.virusres.2016.12.014.

\section{References}

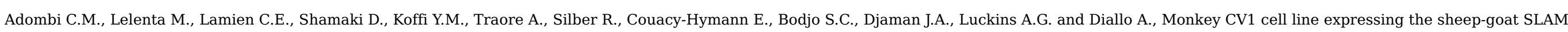


protein: a highly sensitive cell line for the isolation of peste des petits ruminants virus from pathological specimens, J. Virol. Methods 173 (2), $2011,306-313$.

Akira S., Uematsu S. and Takeuchi O., Pathogen recognition and innate immunity, Cell 124 (4), 2006, 783-801.

Albina E., Kwiatek O., Minet C., Lancelot R., Servan de Almeida R. and Libeau G., Peste des Petits Ruminants, the next eradicated animal disease?, Vet. Microbiol. 165 (1-2), 2013, 38-44.

Alexopoulou L., Holt A.C., Medzhitov R. and Flavell R.A., Recognition of double-stranded RNA and activation of NF-kappaB by Toll-like receptor 3, Nature 413 (6857), 2001 , 732-738.

Bailey T.L., Williams N., Misleh C. and Li W.W., MEME: discovering and analyzing DNA and protein sequence motifs, Nucleic Acids Res 34 (Web Server issue), 2006, W369-W373.

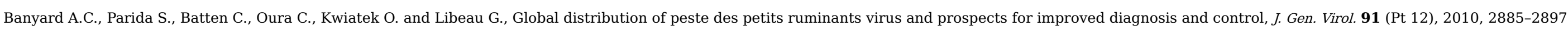

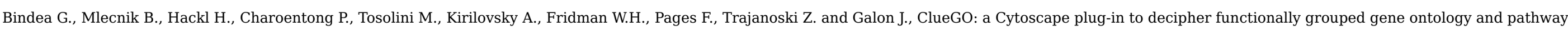

annotation networks, Bioinformatics 25 (8), 2009, 1091-1093.

Bolt G., Berg K. and Blixenkrone-Moller M., Measles virus-induced modulation of host-cell gene expression, J. Gen. Virol. 83 (Pt 5), $2002,1157-1165$.

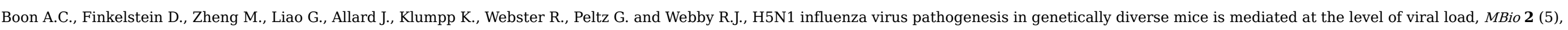
2011.

Boxer E.L., Nanda S.K. and Baron M.D., The rinderpest virus non-structural C protein blocks the induction of type 1 interferon, Virology 385 (1), 2009 , $134-142$.

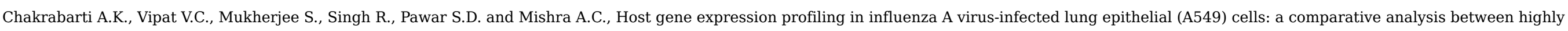
pathogenic and modified H5N1 viruses, Virol. J. 7, 2010, 219.

Collins S.E., Noyce R.S. and Mossman K.L., Innate cellular response to virus particle entry requires IRF3 but not virus replication, J. Virol. 78 (4), $2004,1706-1717$.

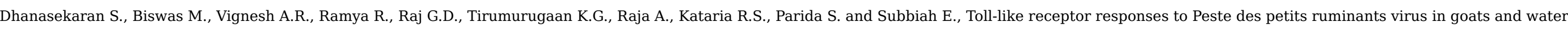
buffalo, PLoS One 9 (11), 2014, e111609.

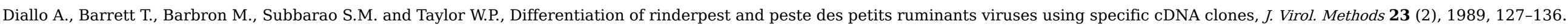

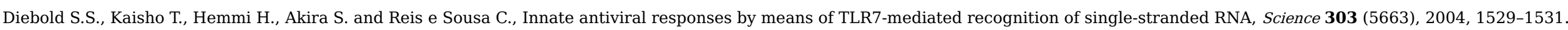

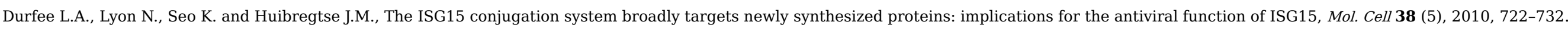

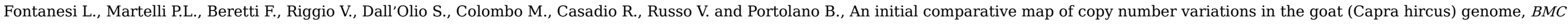
Genomics 11, 2010, 639

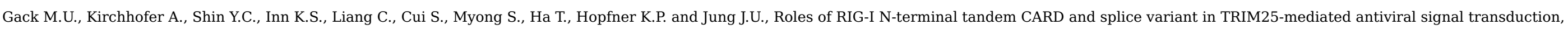
Proc. Natl. Acad. Sci. U. S. A. 105 (43), 2008, 16743-16748.

Girardin S.E., Sansonetti P.J. and Philpott D.J., Intracellular vs extracellular recognition of pathogens-common concepts in mammals and flies, Trends Microbiol. 10 (4), 2002, 193-199.

Gupta S., Stamatoyannopoulos J.A., Bailey T.L. and Noble W.S., Quantifying similarity between motifs, Genome Biol. 8 (2), 2007 , R24.

Harty R.N., Pitha P.M. and Okumura A., Antiviral activity of innate immune protein ISG15, J. Innate Immun. 1 (5), 2009, 397-404.

Hiscott J., Triggering the innate antiviral response through IRF-3 activation, J. Biol. Chem. 282 (21), 2007, 15325-15329.

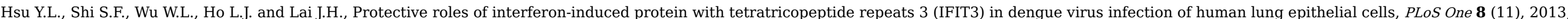
e79518. 
Huang da W., Sherman B.T. and Lempicki R.A., Systematic and integrative analysis of large gene lists using DAVID bioinformatics resources, Nat. Protoc. 4 (1), 2009 , 44-57.

Hummel M., Bonnin S., Lowy E. and Roma G., TEQC: an R package for quality control in target capture experiments, Bioinformatics 27 (9), $2011,1316-1317$.

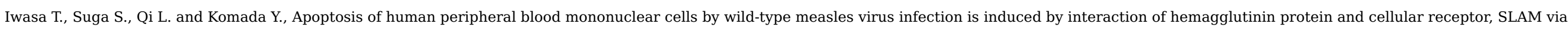
caspase-dependent pathway, Microbiol. Immunol. $\mathbf{5 4}$ (7), 2010, 405-416.

Jensen S. and Thomsen A.R., Sensing of RNA viruses: a review of innate immune receptors involved in recognizing RNA virus invasion, J. Virol. 86 (6), $2012,2900-2910$.

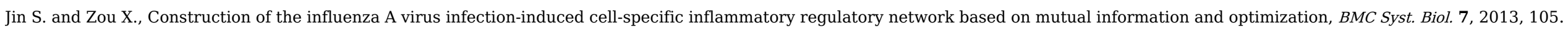

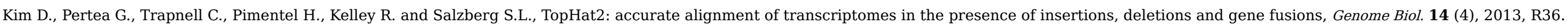

Kohane I.S. and Valtchinov V.I., Quantifying the white blood cell transcriptome as an accessible window to the multiorgan transcriptome, Bioinformatics 28 (4), 2012 , 538-545.

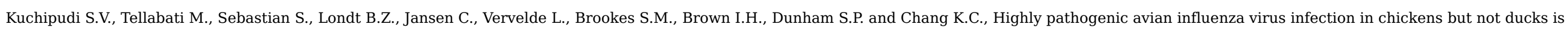
associated with elevated host immune and pro-inflammatory responses, Vet. Res. 45, 2014, 118.

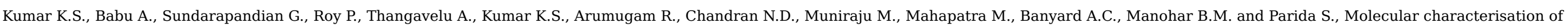
lineage IV peste des petits ruminants virus using multi gene sequence data, Vet. Microbiol. 174 (1-2), 2014a, 39-49.

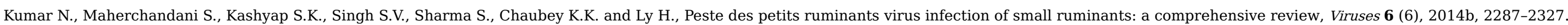

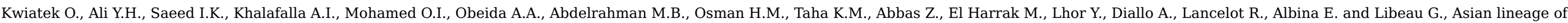
peste des petits ruminants virus, Africa, Emerg. Infect. Dis. 17 (7), 2011, 1223-1231.

Langmead B. and Salzberg S.L., Fast gapped-read alignment with Bowtie 2, Nat. Methods 9 (4), 2012, 357-359.

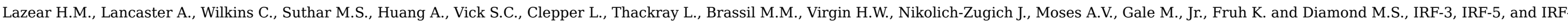
coordinately regulate the type I IFN response in myeloid dendritic cells downstream of MAVS signaling, PLoS Pathog. 9 (1), 2013, e1003118.

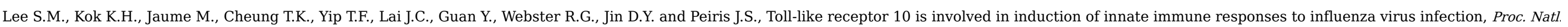
Acad. Sci. U. S. A. 111 (10), 2014, 3793-3798.

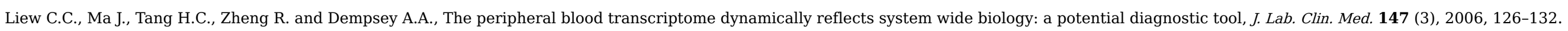

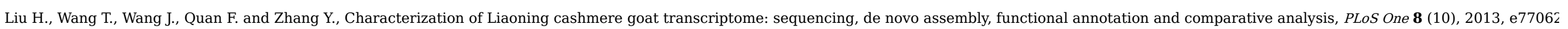

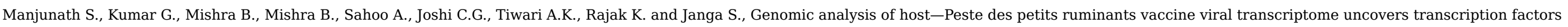
modulating immune regulatory pathways, Vet. Res. 46 (1), 2015, 15.

McNab F.W., Rajsbaum R., Stoye J.P. and O'Garra A., Tripartite-motif proteins and innate immune regulation, Curr. Opin. Immunol. 23 (1), $2011,46-56$.

Nanda S.K. and Baron M.D., Rinderpest virus blocks type I and type II interferon action: role of structural and nonstructural proteins, J. Virol. 80 (15), $2006,7555-7568$.

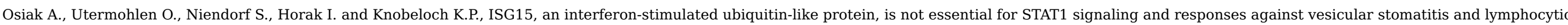
choriomeningitis virus, Mol. Cell. Biol. 25 (15), 2005, 6338-6345.

Patel R.K. and Jain M., NGS QC Toolkit: a toolkit for quality control of next generation sequencing data, PLoS One 7 (2), 2012 , e30619.

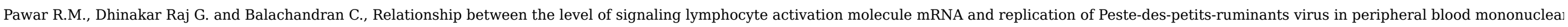
cells of host animals, Acta Virologica. 52 (4), 2008, 231-236. 
Reimand J., Arak T. and Vilo J., g:Profiler-a web server for functional interpretation of gene lists, Nucleic Acids Res. 39 (Web Server issue), 2011 , W307-W315, 2011 update.

Sadler A.J. and Williams B.R., Interferon-inducible antiviral effectors, Nat. Rev. Immunol. 8 (7), 2008, 559-568.

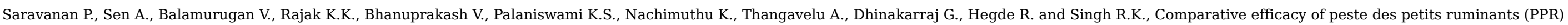
vaccines, Biologicals 38 (4), 2010, 479-485.

Schmid S., Mordstein M., Kochs G., Garcia-Sastre A. and Tenoever B.R., Transcription factor redundancy ensures induction of the antiviral state, J. Biol. Chem. 285 (53), 2010, 42013-42022.

Schmieder R. and Edwards R., Quality control and preprocessing of metagenomic datasets, Bioinformatics 27 (6), 2011, $863-864$.

Schmittgen T.D. and Livak K.J., Analyzing real-time PCR data by the comparative C(T) method, Nat. Protoc. 3 (6), 2008, 1101-1108.

Schneider W.M., Chevillotte M.D. and Rice C.M., Interferon-stimulated genes: a complex web of host defenses, Annu. Rev. Immunol. 32, 2014, 513-545.

Sen G.C. and Sarkar S.N., The interferon-stimulated genes: targets of direct signaling by interferons, double-stranded RNA, and viruses, Curr. Top. Microbiol. Immunol. 316, 2007, 233-250.

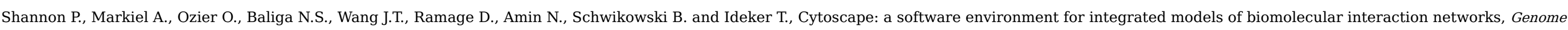
Res. 13 (11), 2003, 2498-2504.

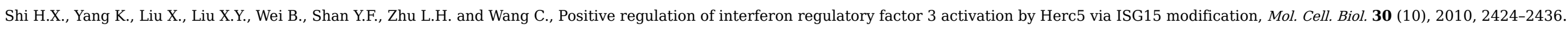

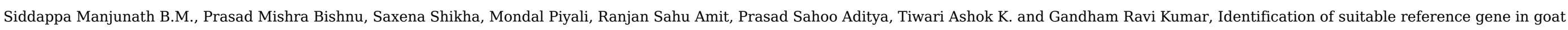
peripheral blood mononuclear cells (PBMCs) infected with peste des petits ruminants virus (PPRV), Livest. Sci. 181, 2015, 15-155.

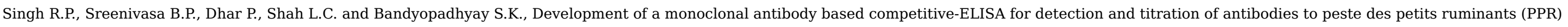
virus, Vet. Microbiol. 98 (1), 2004, 3-15.

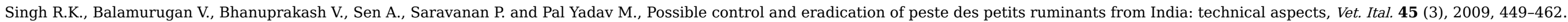

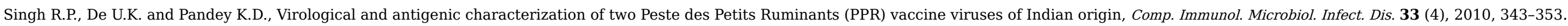

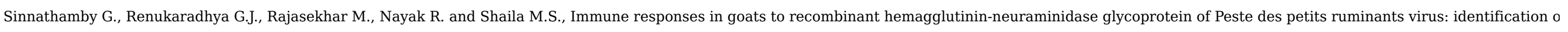
a T cell determinant, Vaccine 19 (32), 2001, 4816-4823.

Stark C., Breitkreutz B.J., Reguly T., Boucher L., Breitkreutz A. and Tyers M., BioGRID: a general repository for interaction datasets, Nucleic Acids Res. 34 (Database issue), 2006, D535-D539.

Tatsuo H. and Yanagi Y., The morbillivirus receptor SLAM (CD150), Microbiol. Immunol. 46 (3), 2002, 135-142.

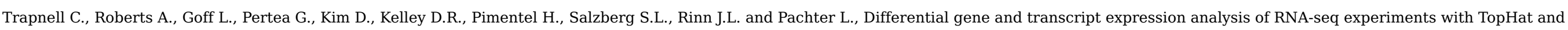
Cufflinks, Nat. Protoc. 7 (3), 2012, 562-578.

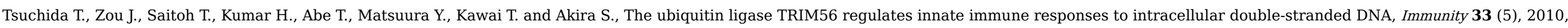
765-776.

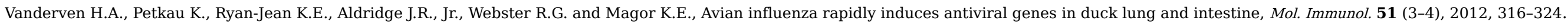

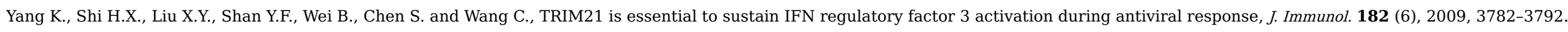

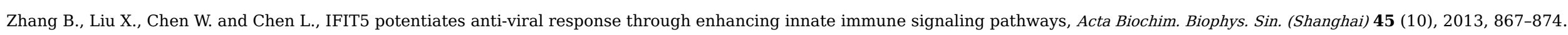

Zhao C., Hsiang T.Y., Kuo R.L. and Krug R.M., ISG15 conjugation system targets the viral NS1 protein in influenza A virus-infected cells, Proc. Natl. Acad. Sci. U. S. A. 107 (5), 2010, $2253-2258$. 


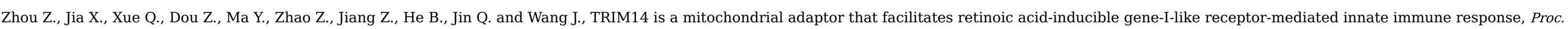
Natl. Acad. Sci. U. S. A. 111 (2), 2014, E245-254.

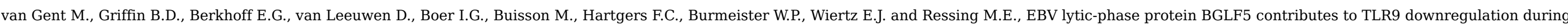
productive infection, J. Immunol. 186 (3), 2011, 1694-1702.

\title{
Appendix A. Supplementary data
}

The following areis Supplementary data to this article:

\author{
$\underline{\text { Multimedia Component } 1}$
}

Highlights

- Temporal dynamics of immune response in PBMCs infected with Sungri/96 vaccine studied

- Differentially expressed genes reflected enrichment of Immune response pathways.

- The upregulation of innate immune genes $=$ IRFs and ISGs identified at early phase.

- Broad-spectrum anti-viral state observed under vaccination.

- Viral transcripts have been quantified in infected PBMCs from the RNA sequencing data.

\section{Queries and Answers}

Query: The author names have been tagged as given names and surnames (surnames are highlighted in teal color). Please confirm if they have been identified correctly. Answer: Yes they are correct

Query: Please check whether all author names and affiliations are correctly linked, and correct if necessary.

Answer: The correct affilation of the co author Y.P Sing is "e" not "1"

Query: Please check the presentation of all affiliations and correct if necessary.

Answer: Incorporate ICAR- at the places mentioned

Query: Please check the address of the corresponding author and correct if necessary.

Answer: it is correct

Query: Please check the presentation of author footnote and correct if necessary.

Answer: correct

Query: Please check the hierarchy of the section headings.

Answer: correct

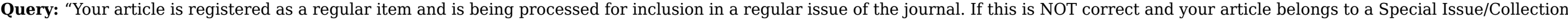




\section{Answer: correct}

Query: Please check the presentation of Section heading "Conflict of interest" and correct if necessary.

Answer: correct

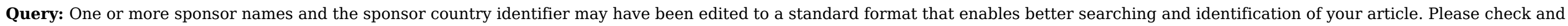
correct if necessary.

Answer: Yes

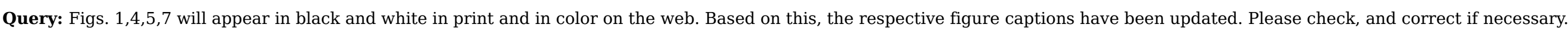

Answer: correct

Query: Please check the presentation of Table 1, and correct if necessary.

Answer: correct 